\title{
EDITORIAL
}

\section{Pulse Oximetry and Predicting Acute Mountain Sickness: Are We Asking the Right Questions?}

\begin{abstract}
"Will I get AMS?" is a question faced by many of those who look after groups in the mountains. After many years of being asked this question, I have developed a well-rehearsed answer. I begin first by giving an estimate of risk. For instance, if we are sitting in one of Lukla's teahouses on the evening of a trek to Mount Everest Base Camp, I will start by admitting that the condition could develop in as many as 30\% during some stage of the trek. ${ }^{1}$ I will then go on to say that there will be some who by simple good fortune will have a lower risk. These will usually be older trekkers who live at moderate altitudes and, despite spending time at high altitude, have never previously suffered from the woes of acute mountain sickness (AMS). Since most of my trekkers tend to be young lowland residents with no previous altitude experience, this information is often met with looks of disappointment. However, before resignation sets in, I focus upon the strategies that I will use to reduce this risk. It is here that I cite the recent Wilderness Medical Society guidelines and stress the importance of a slow, gradual ascent, a light load, and a series of rest days along the route. $^{2}$ At this point the mood normally lifts, but someone will often ask, "Is there anything else that I can do to predict my chances of having AMS?" In recent times, leaders and guides have answered this question by reaching into their pockets and pulling out a pulse oximeter. But does this device offer any help?
\end{abstract}

In 1998, researchers on Denali were the first to explore the link between arterial oxygen saturation $\left(\mathrm{SaO}_{2}\right)$ measurements and the prediction of AMS. The team recruited 102 mountaineers and studied them on arrival at $4200 \mathrm{~m}$ and after a summit attempt. ${ }^{3}$ By establishing a $\mathrm{SaO}_{2}$ cutoff of $84 \%$, researchers were able to retrospectively identify all 21 in whom AMS developed. Unfortunately, an additional 56 climbers who did not have symptoms were also found to have $\mathrm{SaO}_{2}$ of $84 \%$ or less. Similar results were also found in 2010 by a team who studied 83 ascents to a number of different altitudes above $5000 \mathrm{~m} .{ }^{4}$ While a cutoff of $89 \%$ identified all 17 in whom AMS developed, a group of 49 satisfied the $\mathrm{SaO}_{2}$ criteria but the condition never developed. Although these tests were highly sensitive $(100 \%$ and $100 \%$ ), their low levels of specificity ( $31 \%$ and $26 \%$ ) have made many conclude that the pulse oximeter is a poor tool for predicting AMS.

Two studies published in this issue of Wilderness \& Environmental Medicine support this conclusion. In keeping with a number of previous studies, Wagner et $\mathrm{al}^{5}$ were unable to identify any difference in $\mathrm{SaO}_{2}$ measurements between persons who went on to have AMS and those who did not. During an ascent of the popular Mexican volcano Pico De Orizaba (5640 m), the study found that the mean $\mathrm{SaO}_{2}$ measurement of mountaineers at Piedre Grande Hut $(4260 \mathrm{~m})$ only differed by $0.3 \%$ between the 2 groups. Taiwanese researchers, in another study on Jade Mountain (3952 m), examined 833 participants at a number of different sites during an ascent to the summit. ${ }^{6}$ Although $\mathrm{SaO}_{2}$ measurements were lower in participants in whom AMS subsequently developed, this difference was not clinically significant and, importantly, lay within the error range of the pulse oximeter that was used.

Do these results mark the end of predicting AMS with the pulse oximeter? No, not quite. The underlying science remains convincing to many researchers. Look no further than the study by Karinen et $\mathrm{al}^{4}$ for a very clear argument linking a low $\mathrm{SaO}_{2}$ to an increase in AMS susceptibility. Instead, it can be argued that we need to take a few steps back and address some fundamental questions first. Are we using the right device? Do we agree upon the best environment to perform the test? Are we using the pulse oximeter in the right way?

As researchers, we need to start by finding the right pulse oximeter. Under field conditions, such a device must be lightweight, durable, and above all, highly accurate. Large error ranges are simply not good enough when small differences in $\mathrm{SaO}_{2}$ are being sought. Next, we need to agree on a testing protocol that gives the pulse oximeter the best opportunity to work effectively. We have to avoid common sources of error. Fingertips not only need to be free from nail polish and acrylic decorations, but they also have to be warm, still, and hidden from excessive ambient light. Although this environment is difficult to achieve at high altitude, it is vital for any study. Finally, research that relies upon a $\mathrm{SaO}_{2}$ measurement must identify the duration of data collection and the 
a)

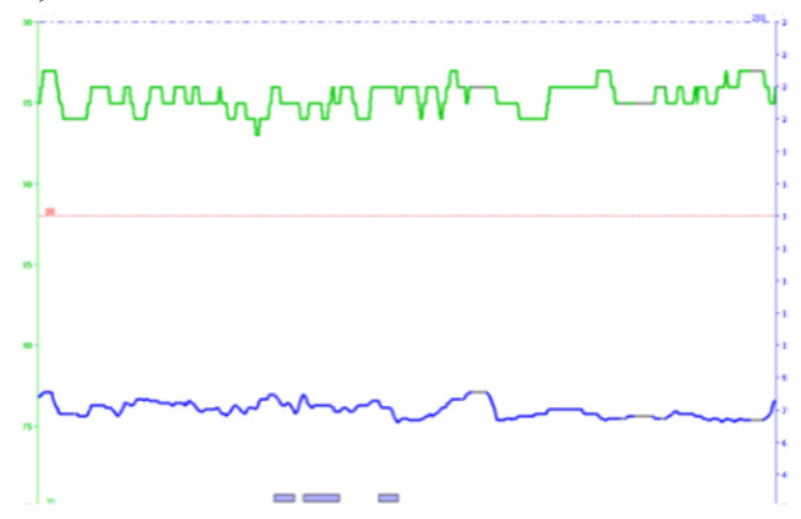

b)

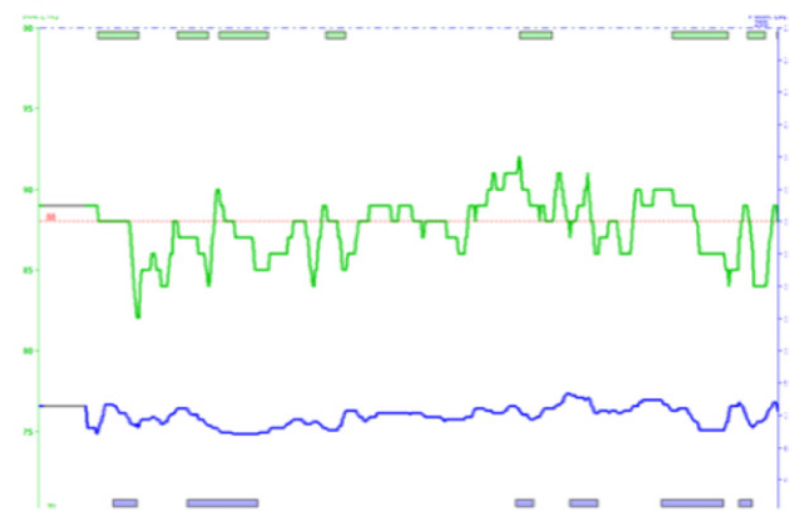

Figure. The resting heart rate (blue) and arterial oxygen saturation $\left(\mathrm{SaO}_{2}\right)$ measurements (green) recorded every second for 10 minutes at a) sea level and at b) Namche Bazaar (3500 m). Each recording was obtained while sitting upright in silence after 15 minutes of rest. The range of $\mathrm{SaO}_{2}$ measurements in this volunteer is considerably greater at high altitude.

activities that are undertaken during it. Given the steepness of the oxyhemoglobin dissociation curve at high altitude, small changes in arterial $\mathrm{Po}_{2}$ can result in large fluctuations in $\mathrm{SaO}_{2}$ (Figure). Who hasn't sat in the mess tent and hyperventilated with a pulse oximeter on their finger? Therefore, is a single resting measurement reliable enough to provide a true reflection of an individual's $\mathrm{SaO}_{2}$ ? Portable devices are now available that continuously measure $\mathrm{SaO}_{2}$ for hours or days at a time. Would measurements during periods of sleep, rest, and exercise provide a better reflection of oxygenation and, therefore, predict AMS with greater accuracy?
Once these fundamental questions have been addressed, it will then be possible to determine whether pulse oximetry can predict AMS. If a more sensitive and specific method is identified, we must then address the most important question of all: what do we do with the information? Should persons with a low $\mathrm{SaO}_{2}$ take an extra rest day? Or could it finally mean that prophylactic drugs like acetazolamide will be targeted toward persons who really need them?

Clearly, there are many questions to address. However, given that portable pulse oximeters are here to stay, we owe it to all those who venture to altitude to try to identify what the role of these devices should be. Here, at Wilderness \& Environmental Medicine, we are keen to play our part. Those asking questions will always have a voice in this journal!

Jeremy S. Windsor, MB, ChB

Mountain Medicine Section Editor

Wilderness \& Environmental Medicine

The Centre for Space, Altitude and Extreme

Environment Medicine

University College

London, UK

\section{References}

1. Basnyat B, Lemastar J, Litch JA. Everest or bust: a cross sectional, epidemiological study of acute mountain sickness at $4243 \mathrm{~m}$ in the Himalayas. Aviat Space Environ Med. 1999;70:867-873.

2. Luks AM, McIntosh SE, Grissom CK, et al. Wilderness Medical Society consensus guidelines for the prevention and treatment of acute altitude illness. Wilderness Environ Med. 2010;21:146-155.

3. Roach RC, Greene ER, Schoene RB, Hackett PH. Arterial oxygen saturation for prediction of acute mountain sickness. Aviat Space Environ Med. 1998;69:1182-1185.

4. Karinen HM, Peltonen JE, Kähönen M, Tikkanen HO. Prediction of acute mountain sickness by monitoring arterial oxygen saturation during ascent. High Alt Med Biol. 2010; 11:325-332.

5. Wagner DR, Knot JR, Fry JP. Oximetry fails to predict acute mountain sickness or summit success during a rapid ascent to 5640 meters. Wilderness Environ Med. 2012;23: 114-121.

6. Chen HC, Lin WL, Wu JY, et al. Change in oxygen saturation does not predict acute mountain sickness on Jade Mountain. Wilderness Environ Med. 2012;23:122-127. 